\title{
LONELINESS, SOCIAL INTERACTIONS AND SENSE OF HUMOR. A QUANTITATIVE STUDY COMPARING ROMANIAN STUDENTS AND OLDER ADULTS
}

\author{
Ioana Schiau \\ National University of Political Studies and Public Administration, Bucharest, Romania \\ ioana.schiau@comunicare.ro
}

\begin{abstract}
The current study explores social and emotional loneliness, social interactions and humor in a sample of Romanian students, departing from the Schiau 2016 study that found the production and social use of humor to be correlated to a reduced social loneliness in a sample of Romanian older adults. Studies indicate that loneliness can be experienced at any age, and that humor can act as a coping mechanism with life's difficulties, triggering positive emotions. The current study replicates findings in the literature, indicating that younger adults experience less loneliness than older adults, and use more humor than older adults. Young women in the sample had a significantly more positive attitude towards humor than the men. This study has useful implications for a number of fields, including the economic and marketing sectors. The current student population represents a growing market, and studies indicate that the use of humor by service providers may intervene with any negative feelings that could cause clients to withdraw their engagement and cooperation in the service endeavor (Locke, 1996). Therefore, we argue that, for the retail and service sector, it is important to understand the different approach towards humor by the different age and gender groups discussed in this study.
\end{abstract}

Keywords

social and emotional loneliness; humor; young adults; older adults; Romania

\section{JEL Classification}

I31

\section{Introduction}

The current study departs from the study by Schiau (2016) that found the use of humor in interpersonal communication to be correlated with a reduced sense of social loneliness in a sample of Romanian older adults. Humor can function as a coping mechanism, helping individuals adapt to various situations and difficulties, across the age groups, and there are indicators in the literature that humor is experienced and valued differently at different ages. Despite the vast research literature that presents loneliness as one of the principal predicaments of ageing, there is strong evidence that loneliness also affects younger adults. Therefore, replicating the study on a sample of Romanian younger adults will help highlight age differences in terms of loneliness and social network correlates, and can offer new information regarding the impact of humor on perceived sense of loneliness for younger adults. Will we find that young adults who use more humor in their social interactions also report feeling less lonely? How will the levels of loneliness reported by students compare to those reported by older adults?

\section{Literature Review \\ Loneliness and Age}

A look into the classical literature on loneliness cannot overlook the 1973 contribution by Robert Weiss, who distinguished between "the loneliness of social isolation" and 
"the loneliness of emotional isolation", two distinct feelings, brought about by two distinct situations: either the lack of a significant and supportive social network, or the lack of close, intimate relationships. In their seminal 1982 work, Peplau and Perlman theorize that loneliness is due to a discrepancy between the individual's desired relationships and the relationships available to them. Research has revealed that loneliness it can be culture-specific, can depend on socio-economic factors, age and gender.

Although loneliness is experienced among individuals of all ages, certain studies suggest older adults are particularly vulnerable to social loneliness (Donaldson \& Watson, 1996). However, there are studies that point out loneliness is also experienced by other age groups (Victor et al. 2002). A 2001 U.S. study found that young adult and older adult participants did not differ in terms of emotional or social loneliness (Green et al., 2001); A 2004 U.K. study by Berguno, Leroux, McAinsh, and Shaikh found that $80 \%$ of children aged 8-10 reported feeling lonely and isolated at school (2004); In a meta-analysis, Pinquart and Sorensen quote studies that find only 5\% - 15\% of older adult study participants to declare ever feeling lonely (Pinquart \& Sorensen, 2001; see also Prince et al., 1997). Loneliness can also be strongly felt by a student population that has moved away to university and living away from family members (Wilcox et al. , 2005), throughout the process of adjusting to major life changes (Rokach \& Brock, 1997) and dealing with a lack of financial capital (Halleröd \& Larsson, 2008) - all situations characteristic to a young university student population. Clearly, there is evidence in the research literature that the issue of loneliness across the lifespan requires further investigation.

One explanation for these differences is that older adults face an array of difficulties brought on by the ageing process, which are correlated with a sense of loneliness: reduced social activity (Aartsen \& Jylhä, 2011), an absence of friends and family (Savikko, 2008; Drennan et al., 2008), living alone, bereavement and widowhood, and lower education (Tilvis et al. 2011, Aartsen \& Jylhä, 2011). One other perspective is the socioemotional selectivity theory by Carstensen (1995), who argues that older adults focus on the most important of their social interactions and are selectively limiting social interaction to close others.

There are reasons to investigate the presence of loneliness in a Romanian student sample, when taking into consideration the cultural and socio-economic climate of the current sample. A 2011 study found that the reported level of loneliness is consistently higher in Eastern Europe than for Western European and Northern European countries, across all age groups, with the former communist countries having the highest level of loneliness (Yang \& Victor, 2011). This could be explained by the shift in society following the fall of communism. Migration is also a phenomenon that could influence feelings of loneliness across all age groups. According to the official 2011 population census by the Romanian National Statistical Institute, 3.6\% of the total population was living abroad, especially adults of working age, which means that many younger adults may have limited direct contact with their parents who have moved abroad to work.

\section{Humor and its relationship to Age and Loneliness}

Humor has been regarded as a source of positive emotions, which can distract the individual from negative emotions and thus reduce said negative feelings (Samson \& Gross, 2012). Apter and Smith (1977) theorize that humor can help individuals see a situation as less severe, and distance themselves from negative emotions. According to Caron (2002), humor produces the positive emotion of mirth, which helps individuals cope with negative situations. There are several theoretical explanations regarding the way humor can function to reduce negative emotions. One theory is the cognitive distraction hypothesis, which proposes that humorous stimuli reduce the resources that 
individuals allocated towards processing negative emotions (Strick et al., 2009). There is evidence in the research literature that humor is correlated with psychological health and resistance to stress, by enhancing perceived social support, according to the study by Martin, Puhlik-Doris, Larsen, Gray, and Weir (2003). Individuals with a greater sense of humor also seem to be more socially competent, as studies by Bell, McGhee, and Duffey (1986), Kuiper, Grimshaw, Leite, and Kirsh (2004) and Yip and Martin (2006) indicate.

The research literature also found age-specific differences in the use and appreciation of humor, which account for the relevance of investigating an age divide in terms of humor in Romania. Thorson, Powell, Sarmany-Schuller, and Hampes, for example found that younger subjects generally score higher than older individuals on humor scales (1997). Ruch and colleagues, found that younger adults laugh more often and more easily than older adults (2010), appreciate verbal humor more and show more appreciation for everyday humor, as indicated in the study by Proyer, Ruch, and Müller (2010).

The current study aims to identify age-related differences in the use of humor, by comparing the findings to those obtained in a methodologically-identical study, carried out using Romanian older adult participants (Schiau, 2016). Since this previous study found that humor can function as a mechanism to reduce social loneliness, the current study proposes to investigate the relationship between humor and loneliness in the same cultural space, but for younger adults.

Ruch, Proyer and Weber propose that findings on the use of humor for older adults could enable practitioners and professionals working with this age group to enrich daily communication (Ruch et al., 2010). Moreover, loneliness has been shown to be correlated with a lower income and weaker purchasing power for goods and services (Halleröd \& Larsson, 2008). Therefore, we argue that a good awareness of patterns of interpersonal communication and socio-emotional indicators for age and gender groups could positively impact the retail and service sectors.

\section{Methodology}

\section{Participants and Procedure}

Self- administered questionnaires were used on a sample of students aged 18 to 30 ( $N$ $=197$ ). A sample of 25 men (mean age $=21, S D=2.53$ ) and 170 women (mean age $=$ $21, S D=1.87$ ) filled in the survey; two respondents did not indicate gender. The survey contained the Multidimensional Sense of Humor Scale (Thorson \& Powell, 1993), the Social and Emotional Loneliness Scale for Adults (diTomaso, Brannen \& Best, 2004). Participants also reported the frequency of interactions with family and friends and filled in socio-demographic data. For the purpose of comparison, the study used of the database for the Schiau (2016) study, which used the same methodology on a sample of Romanian older adults $(N=83 ; 25$ older men, mean age $=67, S D=6,13 ; 58$ older women, mean age $68, S D=6,78)$.

\section{Measures and Validation}

Use of humor in interpersonal relationships. The Multidimensional Sense of Humor Scale (MSHS Thorson \& Powell, 1993) was used to assess participants' self-evaluation on the use of humor in interpersonal relationships. Sample items are "Other people tell me that I say funny things"; "Humor helps me cope"; "I like a good joke"; I can say things in such a way as to make people laugh", and participants indicated on a 5-point Likert scale how well each of the statements described them; from 1 (not at all) to 5 (very mисh). We used a back translated Romanian version of the MSHS, previously translated and validated on a population of Romanian older adults in a study by Schiau 
(2016). A principal components factor analysis was conducted, and produced four dimensions. However, items loaded differently on the four factors than they did in the original English language instrument (Thorson \& Powell, 1993), and also differently from the sample of Romanian older adults (Schiau, 2016), suggesting an age-specific difference in the way humor is used and assessed. The four resulting factors were labeled Production and Social Use of Humor (Cronbach's Alpha = .92); Adaptive Humor (Cronbach's Alpha= .81), Positive Attitude towards Humor (Cronbach's Alpha $=.61$ ) and Negative Attitude towards Humor (Cronbach's Alpha= .63). The overall reliability for the MSHS total scale was Cronbach's Alpha $=.87$

Sense of loneliness. This was measured by using the Social and Emotional Loneliness Scale - Short version (SELSA-S, diTomaso, Brannen \& Best, 2004), an instrument that originally contains three subscales, the Social loneliness subscale, Emotional Loneliness Family subscale and Emotional Loneliness Romantic; the last subscale was not used in the current study. Sample items are "I feel part of a group of friends"; "My friends understand my motives and reasoning"; "My family really cares about me"; "I feel close to my family" and items were rated on a 7-point Likert scale that ranged from 1 (strongly disagree) to 7 (strongly agree). The scale was validated for the Romanian student population by using a back translated version. The items loaded on the two factors exactly as in the original English language scale, and the translated instrument showed very good reliability, the Social Loneliness subscale having Cronbach's Alpha $=.81$ and the Emotional Loneliness Scale having Cronbach's Alpha=.89.

Frequency of social interactions. This was assessed by asking participants to rate their frequency of interactions separately for family and friends (i.e.: "How often do you see your family members?"), using a 5-point Likert-type scale: from 1(every day) to 5 (once every few months or more rarely than this). Participants also rated the quality/meaningfulness of the time spent with family and respectively friends (i.e. "How often do you have positive meaningful interactions with your family - for example, enjoyable conversations, common activities, sharing feelings?"), on a similar answer scale.

\section{Results and Discussion}

\section{The Experience of Loneliness}

Students in our sample reported rather low levels of loneliness, but experienced more social loneliness (mean score $=2.05, \mathrm{SD}=1.13$ ), than emotional loneliness (mean score $=1.72, \mathrm{SD}=1.26$ ). This could be interpreted as a sign that the adjustment to university life, to a completely new social network of friends and colleagues, perhaps to a new city, poses difficulties to students and results in higher levels of social loneliness; we could speculate that, in this situation, students come to rely more on their family ties, resulting in lower levels of emotional (family) loneliness. When investigating gender differences in terms of loneliness, the results show that women in the sample feel both less social and less emotional loneliness than the men; however, the difference is not statistically significant.

Individuals in the sample who felt less social loneliness were those who had more frequent interactions with friends $(\mathrm{r}(194)=.18, \mathrm{p}=.008)$ and more frequent meaningful interactions with friends $(\mathrm{r}(194)=.34, \mathrm{p}=.00)$. This is according to the theory of loneliness (Weiss, 1973), and also shows that the research instruments employed by the study correctly measure the concept of loneliness.

When comparing the results to those obtained on a sample of older adults, the Romanian students reported significantly less social loneliness (Mean score for students $=2.05, S D=1.13$; Mean score for older adults $=2.63, S D=1.09 ; t=-4.05, d f=160$, $p=.00$ ) and significantly less emotional loneliness (Mean score for students $=1.72, S D=$ 1.26; Mean score for older adults $=2.05, S D=1.50 ; t=-1.90, d f=274, p=.05$ ) than the Romanian older adults. Compared to older adults, younger adults also reported 
significantly more frequent social interactions with friends, and more frequent meaningful interactions with friends. These finding are in line with other research studies that found older adults to report significantly higher levels of loneliness. It could be speculated that, in Romania, this is an effect partly due to the societal shift following the fall of communism, such as Schwartz, Bardi, and Bianchi indicate (2000). This will have left individuals in a state of mistrust towards others and having to adapt to new relationship patterns, suggest studies such as that of Schwarzer, Hahn, and Schröder (1994). These changes may have primarily affected current older adults, who were in their prime in 1989, rather than younger adults such as those in the current sample, who were born after 1989 .

\section{The Use of Humor in Interpersonal Communication}

Analyzing the results obtained on the Multidimensional Sense of Humor Scale, the four dimensions of the humor scale correlate in an intuitive manner, suggesting that the instruments measure the concepts correctly. The Production and Social Use of Humor scale correlated with a Positive Attitude towards Humor $(\mathrm{r}=.35, \mathrm{~N}=193, \mathrm{p}=.00)$ and with Adaptive Humor $(\mathrm{r}(193)=.79, \mathrm{p}=.00)$, the latter two dimensions also correlating positively amongst themselves $(\mathrm{r}(193)=.39, \mathrm{p}=.00)$. Intuitively, a Negative Attitude towards Humor is correlated negatively with a Positive Attitude towards Humor $(\mathrm{r}(193)=-.23, \mathrm{p}=.001)$.

We analyzed the gender differences for the student sample and found no statistically significant difference in the scores men and women obtained on the Production and Social Use of Humor and Adaptive Humor dimensions, the mean scores being almost identical. However, one interesting result was that men had a significantly lower mean score on the Positive Attitude towards Humor scale ( $t=-3.6, d f=191, p=.03)$, and a significantly higher mean score on the Negative Attitude towards Humor scale $(t=-2.6$, $d f=33.19, \mathrm{p}=.01)$. Young women in our sample generally view humor more positively than young men.

When comparing the results to those obtained on a sample of older adults, for the dimensions labeled Production and Social Use of Humor and Adaptive Humor, the Romanian students obtained a significantly higher score on the former subscale, indicating that they use more humor in their interpersonal communication (Mean score for students $=3.54, S D=.78$; Mean score for older adults $=3.12, S D=.99 ; t=-3.74, d f=$ $274, p=.00)$. This is in line with other studies, including by Thorson, Powell, SarmanySchuller and Hampes that found older adults to score lower on the MSHS. This is a difficult result to interpret, being that humor has also been revealed to be able to help older adults cope with the difficulties of the ageing process (Dziegielewski et al. 2004) and that older adults are found to generally be more inclined towards positive affect (Ready et al., 2011). Because we lack comparative trans-national studies regarding humor use and appreciation scores, it is difficult to attribute this finding to a cultural or psycho-social context.

However, this result is relevant for the retail and service sectors, which could keep this finding in mind when elaborating protocols for communication with younger or older consumers. Previous studies found that the use of humor by service providers may foster client engagement and cooperation in the service endeavor (Locke, 1996), and the findings of the current study indicate that younger adults are more prone to making use of humor in their interpersonal communication. This could be argued to be true for their transactions and purchase activities as well. We therefore recommend the use of humorous communication as a strategy more likely to succeed with younger adults.

\section{The Relation between Humor and Loneliness}


When seeking to investigate the connection between humor and a sense of loneliness, we discovered that only a Positive Attitude towards Humor correlated negatively with the sense of Social Loneliness, $(\mathrm{r}(192)=-.15, \mathrm{p}=.03)$; no correlation was found between humor and Emotional Loneliness. However, when controlling for gender, we found that this correlation was strongest for the women in our sample, and was much weaker and not significant for the men. This is an interesting finding, suggesting that women who have a positive attitude towards humor and appreciate its positive effects in social interactions can also experience a reduced sense of loneliness.

The findings were compared with those obtained on the sample of Romanian older adults. For Romanian older adults, a significant correlation was only for older women, and not for older men: the Production and Social Use of Humor was correlated to a reduced sense of Social Loneliness. However, for a younger category of adults, it seems that it is not the actual use of humor in interpersonal communication that can help ease a sense of loneliness. The less lonely individuals in the sample were those who had a generally positive attitude towards humorous interactions, jokes and humorous people. It could be speculated that women who appreciate humor are prone to have a positive outlook on life, and could therefore feel more optimistic about their social network and less lonely.

The connection between humor and frequency of interactions with friends and family was also investigated. There were significant correlations between the Production and Social Use of Humor and the frequency of interactions with friends $(r(192)=.24, p=$ $.001)$, and the frequency of meaningful interactions with friends $(r(192)=.27, p=.00)$. Adaptive Humor was significantly correlated in a similar manner, to the same two factors: frequency of interactions with friends $(\mathrm{r}(192)=.25, \mathrm{p}=.001)$, and the frequency of meaningful interactions with friends $(\mathrm{r}(192)=.17, \mathrm{p}=.01)$. This suggests that people in the sample who use more humor in their interactions and who can use humor as a mechanism to cope with different situations are also the individuals who can maintain a strong social network, providing them with support and companionship.

\section{Conclusions}

The current study, despite the specific limitations of a quantitative approach based on self-report, has managed to bring a small, but interesting contribution to the literature on loneliness and humor in older age. Firstly, the study replicated the findings of previous research, indicating that younger adults report experiencing less loneliness than older adults do, and report using more humor than older adults do. Young adults also indicated that they meet their friends more often, and have more frequent meaningful interactions with them.

Further research on this topic could explore the main factors that trigger feelings of social loneliness, and should consider the possibility of a socioemotional regulation process, where older adults willingly decide to narrow down their social network and focus on their most important and close relationships.

Interestingly, for a Romanian student population, the use of humor in interpersonal communication did not correlate with a reduced sense of social loneliness, as in the model found for Romanian older women. Rather, for students in our sample, it was the positive attitude towards humor that was connected to a reduced sense of social loneliness (the correlation being much stronger for women); participants with a more positive attitude towards humor were also those who had more frequent meetings and more frequent meaningful interactions with friends.

There are grounds to speculate here that humor is experienced and appreciated differently by Romanian young men and women, with young women having a significantly more positive attitude towards humor. This issue requires further investigation. 
Consequently, a number of recommendations can result from the present study, to aid professionals in economic, retail and service sectors, as well as those working in marketing and advertising, when it comes to the communication strategies and protocols to be observed for specific age and gender groups.

Firstly, there are indications in the study that Romanian young women have a more positive attitude towards humor than young men do - this could be an interesting insight for the advertising industry, where advertisements targeted at women rarely make use of humor. Unique selling propositions directed at women consumers should take into account their willingness to engage with humorous messages. Moreover, it seems that younger adults make more use of humor in their interpersonal communication than older adults do, according to the own reports of the participants in this study. This would lead to the assumption that humorous communication is a strategy that better fits young customers and consumers. This is a matter that should be researched further in subsequent studies, targeted specifically at investigation the reception and appreciation of humorous communication in purchasing contexts, client service protocols, or in advertising.

As expected and suggested by previous research studies, the level of loneliness reported by older adults was higher than indicated by younger adults. This could also be due to cohort effects, where the current cohort of older adults have experienced life during the communist system. This has led to a mistrust in social relations (Schwarzer et al., 1994) and, subsequently, increased levels of loneliness. And, most significantly for the economic sector, this age cohort was socialized with a particular set of social and communication protocols, and was deeply reliant on alternative economic systems (e.g. the black market or unofficial trades with other individuals). The design of a communication protocol for this age cohort should take these factors into account.

\section{References}

Apter, M. J., \& Smith, K. C. P. (1977), Humor and the theory of psychological reversals, In A. J. a. F. Chapman, \& H. C. Foot (Ed.), It's a funny thing humor (pp. 182-210), Oxford: Pergamon Press.

Aartsen, M., \& Jylhä, M. (2011), Onset of loneliness in older adults: results of a 28 year prospective study. Social, Behavioural and Health Perspectives, 8(1), 31-38. http://doi.org/10.1007/s10433-011-0175-7.

Bell, N. J., McGhee, P. E., \& Duffey, N. S. (1986), Interpersonal competence, social assertiveness and the development of humour. British Journal of Developmental Psychology, 4(1), 51-55. http://doi.org/10.1111/j.2044-835X.1986.tb00997.x,

Berguno, G., Leroux, P., McAinsh, K., \& Shaikh, S. (2004), Children's Experience of Loneliness at School and Its Relation to Bullying and the Quality of Teacher Interventions. Qualitative Report, 9(3), 483-499.

Caron, J. E. (2002), From ethology to aesthetics: Evolution as a theorical paradigm for research on laughter, humor, and other comic phenomena. Humor: International Journal of Humor Research, 15 (3), 245-281.

Carstensen, L. L. (1995). Evidence for a life-span theory of socioemotional selectivity. Current Directions in Psychological Science, 4, 151-156.

DiTommaso, E., Brannen, C., \& Best, L. A. (2004), Measurement and Validity Characteristics of the Short Version of the Social and Emotional Loneliness Scale for Adults. Educational and Psychological Measurement, 64(1), 99-119. http://doi.org/10.1177/0013164403258450.

Donaldson, J. M., \& Watson, R. (1996), Loneliness in elderly people: an important area for nursing research. Journal of Advanced Nursing, 24(5), 952-959. http://doi.org/10.1111/j.1365-2648.1996.tb02931.x. 
Drennan, J., Treacy, M., Butler, M., Byrne, A., Fealy, G., Frazer, K., \& Irving, K. (2008), The experience of social and emotional loneliness among older people in Ireland. Ageing and Society, 28(8), 1113-1132. http://doi.org/10.1017/S0144686X08007526.

Dziegielewski, S. F., Jacinto, G. A., Laudadio, A. and Legg-Rodriguez, L. 2004, Humor: an essential communication tool in therapy. International Journal of Mental Health, 32, 3, 74-90.

Green, L., Richardson, D., Lago, T., \& Schatten-Jones, E. (2001), Network Correlates of Social and Emotional Loneliness in Young and Older Adults. Personality and Social Psychology Bulletin, 27(3), 281-288.

Halleröd, B., \& Larsson, D. (2008), Poverty, welfare problems and social exclusion. International Journal of Social Welfare, 17(1), 15-25.

Koc, Z. (2012), Determination of older people's level of loneliness. Journal of Clinical Nursing, 21 (21-22), 3037-3046.

Kuiper, N. A., Grimshaw, M., Leite, C., \& Kirsh, G. (2004), Humor is not always the best medicine: Specific components of sense of humor and psychological well-being. Humor: International Journal of Humor Research, 17, 135168. http://dx.doi.org/10.1515/humr.2004.002.

Locke, K. (1996), A Funny Thing Happened! The Management of Consumer Emotions in Service Encounters. Organization Science, 7(1), 40-59.

Martin, R. A., Puhlik-Doris, P., Larsen, G., Gray, J., \& Weir, K. (2003), Individual differences in uses of humor and their relation to psychological well-being: Development of the humor styles questionnaire. Journal of Research in Personality, 37, 48-75. http://dx.doi.org/10.1016/s0092-6566(02)00534-2.

Official Population Census. (2011), Romanian National Statistical Institute. Retrieved from http://www.recensamantromania.ro/wp content/uploads/2013/07/REZULTATE-DEFINITIVE-RPL_2011.pdf

Peplau, L. A., Miceli, M., \& Morasch, B. (1982), Loneliness and Self-Evaluation. In L. A. Peplau \& D. Perlman (Eds.), Loneliness: A Sourcebook of Current Theory, Research and Therapy (135-151). New York, U.S.A.: Wiley and Sons.

Pinquart, M., \& Sorensen, S. (2001), Influences on Loneliness in Older Adults: A MetaAnalysis.Basic and Applied Social Psychology, 23(4), 245-266.

Prince, M. J., Harwood, R. H., Blizard, R. A., \& Thomas, A. (1997), Social support deficits, loneliness and life events as risk factors for depression in old age: The Gospel Oak Project VI. Psychological Medicine, 27, 323-332.

Proyer, R., Ruch, W., \& Muller, L. (2010), Sense of humor among the elderly Findings using the German version of the SHS. Z. Gerontol. Geriatr., 43(1), 19-24. http://doi.org/10.1007/s00391-009-0082-0.

Ready, R., Vaidya, J., Watson, D., Latzman, R., Koffel, E., \& Clark, L. (2011), Age-group differences in facets of positive and negative affect. Aging \& Mental Health, 15(6), 784-795.

Rokach, A., \& Brock, H. (1997), Loneliness and the Effects of Life Changes. The Journal of Psychology, 131(3), 284-298.

Ruch, W., Proyer, R., \& Weber, M. (2010a), Humor as a character strength among the elderly Empirical findings on age-related changes and its contribution to satisfaction with life. Z. Gerontol. Geriatr., 43(1), 13-18. http://doi.org/10.1007/s00391-009-0090-0.

Samson, A. C., \& Gross, J. J. (2012), Humour as emotion regulation: The differential consequences of negative versus positive humour. Cognition and Emotion, 26(2), $375-384$.

Savikko, N. (2008), Loneliness of Older People and Elements of an Intervention for its Alleviation. University of Turku, Turku. 
Schiau, I. Humor, Loneliness and Interpersonal Communication: A Quantitative Study of Romanian Older Adults. Romanian Journal of Communication and Public Relations 18.1 (2016): 89-106.

Schwartz, S. H., Bardi, A., \& Bianchi, G. (2000), Value adaptation to the imposition and collapse of communist regimes in East-Central Europe. In J. Duckitt \& S. A. Renshon (Eds.), Political psychology: Cultural and cross-cultural perspectives (pp. 217-235), New York: Macmillan.

Schwarzer, R., Hahn, A., \& Schröder, H. (1994), Social integration and social support in a life crisis: Effects of macrosocial change in East Germany. American Journal of Community Psychology, 22, 685-706.

Strick, M., Holland, R. W., Van Baaren, R. B., \& Van Knippenberg, A. (2009), Finding comfort in a joke: Consolatory effects of humor through cognitive distraction. Emotion, 9(4), 574-578.

Thorson, J. A., \& Powell, F. C. (1993), Development and validation of a Multidimensional Sense of Humor Scale. Journal of Clinical Psychology, 49(1), 13-23.

Thorson, J., Powell, F., Sarmany-Schuller, I., \& Hampes, W. (1997), Psychological health and sense of humor. Journal of Clinical Psychology, 53(6), 605-19.

Tilvis, R. S., Laitala, V., Routasalo, P. E., \& Pitkälä, K. H. (2011), Suffering from Loneliness Indicates Significant Mortality Risk of Older People. Journal of Aging Research, 2011, 1-5. http://doi.org/10.4061/2011/534781.

Victor, Scambler, Shah, Cook, Harris, Rink, \& De Wilde. (2002), Has loneliness amongst older people increased? An investigation into variations between cohorts. Ageing And Society, 22, 585-597.

Weiss, R. S. (1973), Loneliness : the experience of emotional and social isolation. Cambridge, Mass.: Cambridge, Mass.

Wilcox, P., Winn, S., \& Fyvie-Gauld, M. (2005), 'It was nothing to do with the university, it was just the people': The role of social support in the first-year experience of higher education. Studies in Higher Education, 30(6), 707-722.

Yang, K. \& Victor, C. (2011), Age and loneliness in 25 European nations. Ageing and Society, 31(8), 1368-1388, http://doi.org/10.1017/S0144686X1000139X.

Yip, J.A., Martin, R.A. (2006), Sense of humor, emotional intelligence, and social competence. Journal of Research in Personality 40 1202-1208, http://dx.doi.org/10.1016/j.jrp.2005.08.005 